\title{
ON THE TAIL OF JONES POLYNOMIALS OF CLOSED BRAIDS WITH A FULL TWIST
}

\author{
ABHIJIT CHAMPANERKAR AND ILYA KOFMAN
}

(Communicated by Daniel Ruberman)

\begin{abstract}
For a closed $n$-braid $L$ with a full positive twist and with $\ell$ negative crossings, $0 \leq \ell \leq n$, we determine the first $n-\ell+1$ terms of the Jones polynomial $V_{L}(t)$. We show that $V_{L}(t)$ satisfies a braid index constraint, which is a gap of length at least $n-\ell$ between the first two nonzero coefficients of $\left(1-t^{2}\right) V_{L}(t)$. For a closed positive $n$-braid with a full positive twist, we extend our results to the colored Jones polynomials. For $N>n-1$, we determine the first $n(N-1)+1$ terms of the normalized $N$-th colored Jones polynomial.
\end{abstract}

\section{INTRODUCTION}

The tail (resp. head) of a polynomial will denote the sequence of its lowest (resp. highest) degree terms, up to some specified length. In this note, we precisely determine the tail of the Jones polynomial for a closed $n$-braid with a full positive twist and with up to $n$ negative crossings. We also precisely determine the tails of the colored Jones polynomials for a closed positive $n$-braid with a full positive twist.

It is natural to consider quantum and geometric invariants of links that are closed braids with a full twist. For example, Lorenz links, all of which are closed positive braids with a full twist, dominate the census of the simplest hyperbolic knots, and their Jones polynomials are relatively simple [2, 5. The full twist arises as \pm 1 Dehn surgery on the braid axis, considered as an augmented unknot in $S^{3}$. Hence, adding full twists is a natural geometric operation on links. On the other hand, the full twist is in the center of the braid group, so its image in any irreducible representation is a scalar. Most known closed formulas for Jones polynomials of infinite link families essentially rely on this fact.

For any closed braid, we showed in [4] that after sufficiently many full twists on a subset of strands, the coefficient vector for any colored Jones polynomial decomposes into fixed blocks, separated by blocks of zeros that increase by a constant length for every twist. So once the nonzero blocks separate, they simply move apart unchanged with every additional full twist. In Theorem 1.1 below, we completely determine the first block for full twists on all strands. In this case, the first block separates after only one full twist.

Dasbach and Lin [6] showed that for alternating knots, and more generally $A-$ adequate knots, the first three coefficients in the tail of the normalized $N$-th colored

Received by the editors April 5, 2011 and, in revised form, October 15, 2011.

2010 Mathematics Subject Classification. Primary 57M25.

Both authors gratefully acknowledge support by the NSF, Simons Foundation, and PSCCUNY. 
Jones polynomials are independent of the color for $N \geq 3$. In Corollary 1.4 for $N>n-1$, we determine the tail of length $n(N-1)+1$ for the normalized $N$-th colored Jones polynomial of closed positive braids with a full twist. More precisely, these coefficients consist of $n-1$ blocks of length $N+1$, where each block is a \pm 1 followed by $N$ zeros. Armond [1] proved related results about tails of colored Jones polynomials for any closed positive braid.

Theorem 1.3 implies that given $M \geq 2$, for all colors $N \geq M$, the coefficients of the tail of length $M$ stabilize up to sign. Garoufalidis [10 proved that any finite-depth coefficient of the colored Jones polynomial satisfies a linear recursion with constant coefficients. From this point of view, Theorem 1.3 implies that these particular colored Jones coefficients satisfy a trivial linear recursion with constant coefficients.

Dasbach and Lin also showed that the second coefficients of the head and tail together provide a linear bound for the hyperbolic volume of alternating knots. Futer, Kalfagianni and Purcell [8, 9] showed this as well for hyperbolic closed 3braids and many classes of hyperbolic adequate links, but that it fails to hold for other such classes (see [9]). In contrast, the coefficients of the tail of length $N$ as in Theorem 1.3 for closed positive braids with a full twist are all $\{-1,0,1\}$. Moreover, the tail of length $n(N-1)$, as in Corollary 1.4 also has coefficients only $\{-1,0,1\}$. These coefficients and the dependence on the braid index indicate that, for this class of knots, these tails by themselves are unrelated to the hyperbolic volume of the closed braid. For example, 3-braids have unbounded hyperbolic volume [9], and the positive twisted torus knots $T(p, q, 2, s)$ with $p, q>2, s>0$, have bounded hyperbolic volume but unbounded braid index 3 .

To state our main results, we adopt the following standard convention. Let $V_{L}(t)$ denote the Jones polynomial, such that

$$
t^{-1} V_{L_{+}}-t V_{L_{-}}=\left(t^{1 / 2}-t^{-1 / 2}\right) V_{L_{0}} \quad \text { and } \quad V_{\bigcirc}(t)=1 .
$$

Theorem 1.1. Let $\beta^{\prime}$ be an $n$-braid of length $c$ with $\ell$ negative crossings with $0 \leq \ell \leq n$ and $\beta=\Delta_{n}^{2} \beta^{\prime}$, where $\Delta_{n}^{2}$ is the positive full twist in the braid group $B_{n}$. Then

$$
\begin{aligned}
V_{\bar{\beta}}(t) & =(-1)^{n+c+1} t^{\frac{(n-1)^{2}+c-2 \ell}{2}}\left(\frac{1+t^{n-\ell+1} p(\bar{\beta} ; t)}{1-t^{2}}\right) \\
& =(-1)^{n+c+1} t^{\frac{(n-1)^{2}+c-2 \ell}{2}}\left(\sum_{i=0}^{[(n-\ell) / 2]} t^{2 i}+t^{n-\ell+1} q(\bar{\beta} ; t)\right),
\end{aligned}
$$

where $p(\bar{\beta} ; t)$ and $q(\bar{\beta} ; t)$ are polynomials in $t$.

The latter expression gives the tail of $V_{\bar{\beta}}(t)$ of length $n-\ell+1$. An interesting consequence is that the Jones polynomial satisfies a braid index constraint, which is a gap of length at least $n-\ell$ between the first two nonzero coefficients of $\left(1-t^{2}\right) \cdot V_{\bar{\beta}}(t)$. If $\ell=0$ in Theorem 1.1, then $\beta$ is a positive $n$-braid with a full twist. In this case, the MFW inequality [14, 7] is sharp, so the braid index of $\bar{\beta}$ is $n$. However, the gap between the first two nonzero coefficients of $\left(1-t^{2}\right) V_{\bar{\beta}}(t)$ can be more than $n$. For example, if $\beta^{\prime}=\sigma_{2}^{2} \sigma_{1} \in B_{4}$, then $\beta=\Delta_{4}^{2} \beta^{\prime}$ and

$$
V_{\bar{\beta}}(t)=t^{6}+t^{8}+t^{10}+t^{12} \Longrightarrow\left(1-t^{2}\right) V_{\bar{\beta}}(t)=t^{6}-t^{14} \text {. }
$$


Another consequence of Theorem 1.1 is related to a conjecture of V. Jones [12], which remains open in general: The writhe $w(\beta)$, which is the algebraic crossing number of $\beta$, is a topological invariant of $\bar{\beta}$ whenever $n$ is the minimal braid index of $\bar{\beta}$ (see [15). When the MFW inequality is sharp, the Jones conjecture is known to hold, which is the case for positive braids, $\ell=0$. If $\ell=1, \beta$ is conjugate to a positive braid. For $\ell>1$, although we do not know when the MFW inequality is sharp, we can prove the Jones conjecture:

Corollary 1.2. For $\beta$ as in Theorem 1.1 ,

$$
2 \min \operatorname{deg}\left(V_{\bar{\beta}}(t)\right)=w(\beta)-n+1 .
$$

Thus, if $n$ is the minimal braid index of $\bar{\beta}$, then $w(\beta)$ is a topological invariant of $\bar{\beta}$.

Proof. 2 min $\operatorname{deg}\left(V_{\bar{\beta}}(t)\right)=(n-1)^{2}+c-2 \ell=n(n-1)+c-2 \ell-n+1=w(\beta)-n+1$.

Let $J_{N}(L ; t)$ be the colored Jones polynomial of $L$, colored by the $N$-dimensional irreducible representation of $\mathfrak{s l}_{2}(\mathbb{C})$, with the normalization

$$
J_{2}(L ; t)=\left(t^{1 / 2}+t^{-1 / 2}\right) V_{L}(t) \text { and } J_{N}(\bigcirc ; t)=\frac{t^{N / 2}-t^{-N / 2}}{t^{1 / 2}-t^{-1 / 2}}=[N] .
$$

The colored Jones polynomials are weighted sums of Jones polynomials of cablings, and the following formula is given in [13]. Let $L^{(r)}$ be the 0 -framed $r$-cable of $L$; i.e., if $L$ is 0 -framed, then $L^{(r)}$ is the link obtained by replacing $L$ with $r$ parallel copies:

$$
J_{N+1}(L ; t)=\sum_{j=0}^{[N / 2]}(-1)^{j}\left(\begin{array}{c}
N-j \\
j
\end{array}\right) J_{2}\left(L^{(N-2 j)} ; t\right) .
$$

The normalized colored Jones polynomial $J_{N}^{\prime}(L ; t)$ is defined by

$$
J_{N}^{\prime}(L ; t)=\frac{J_{N}(L ; t)}{[N]} \text { and } J_{N}^{\prime}(\bigcirc ; t)=1 .
$$

Theorem 1.3. Let $\beta^{\prime}$ be a positive $n$-braid of length $c$ and $\beta=\Delta_{n}^{2} \beta^{\prime}$, where $\Delta_{n}^{2}$ is the positive full twist in the braid group $B_{n}$. Then

$$
\begin{aligned}
J_{N+1}^{\prime}(\bar{\beta} ; t) & =(-1)^{N(n+c+1)} t^{\frac{N\left((n-1)^{2}+c\right)}{2}}\left(\frac{1+t^{n N+1} p_{N}(\bar{\beta} ; t)}{1-t^{N+1}}\right) \\
& =(-1)^{N(n+c+1)} t^{\frac{N\left((n-1)^{2}+c\right)}{2}}\left(\sum_{i=0}^{n-1} t^{i(N+1)}+t^{n N+1} q_{N}(\bar{\beta} ; t)\right),
\end{aligned}
$$

where $p_{N}(\bar{\beta} ; t)$ and $q_{N}(\bar{\beta} ; t)$ are polynomials in $t$.

Corollary 1.4. If $\beta^{\prime}$ is a positive $n$-braid of length $c$ and $\beta=\Delta_{n}^{2} \beta^{\prime}$, then for $N>n-2$,

$J_{N+1}^{\prime}(\bar{\beta} ; t)=(-1)^{N(n+c+1)} t^{\frac{N\left((n-1)^{2}+c\right)}{2}}\left(\sum_{i=0}^{n-1} t^{i(N+1)}+\{\right.$ terms of degree $\left.\geq n N+1\}\right)$.

Proof. In Theorem 1.3, $\sum_{i=0}^{n-1} t^{i(N+1)}$ and $t^{n N+1} q_{N}(\bar{\beta} ; t)$ can overlap only when $(n-1)(N+1) \geq n N+1$, i.e. when $N \leq n-2$. 
For $N>n-1$, Corollary 1.4 determines the tail of length $n(N-1)+1$ for the normalized $N$-th colored Jones polynomial.

\section{Proof of Theorem 1.1}

Generalizing the well-known formula for torus knots, the Jones polynomial of any torus link $T(p, q)$ is given by the following sum, with $d=\operatorname{gcd}(p, q)$ [11:

$$
V_{T(p, q)}(t)=(-1)^{d+1} \frac{t^{(p-1)(q-1) / 2}}{1-t^{2}} \sum_{i=0}^{d}\left(\begin{array}{l}
d \\
i
\end{array}\right) t^{\frac{p}{d}\left(1+\frac{q}{d} i\right)(d-i)}\left(t^{\frac{q}{d}(d-i)}-t^{1+\frac{q}{d} i}\right) .
$$

For $n=2$, the claim follows from (2.1), which in this case simplifies to

$$
V_{T(2, q)}(t)=(-1)^{q+1} \frac{t^{(q-1) / 2}}{\left(1-t^{2}\right)}\left(1-t^{3}+(-1)^{q}\left(t^{1+q}-t^{2+q}\right)\right) .
$$

Henceforth, let $n>2$. The Jones polynomial $V_{L}(t)$ is obtained from the Kauffman bracket $\langle L\rangle$ by substituting $t=A^{-4}$ and multiplying by $\left(-A^{3}\right)^{-w}$ to adjust for the writhe $w$ of $L$. We will show that the right-most part of the coefficient vector of the Kauffman bracket $\vec{c}=\left\{c_{i} \mid\langle L\rangle=A^{*} \sum c_{i} A^{4 i}\right\}$ has the following form:

$$
* \underbrace{01 \ldots 01}_{n+1} \text { if } n \text { is odd } \quad * \underbrace{-10-1 \ldots 0-1}_{n+1} \text { if } n \text { is even. }
$$

We will call this part of the Kauffman bracket the gap block. Multiplying by $1-t^{2}=$ $1-A^{-8}$, the coefficient vector changes as follows:

$$
\begin{array}{rlrl} 
& \text { Case: } n \text { odd } & \text { Case: } n \text { even } \\
\left((-1)^{n+1}\langle L\rangle\right) & * * * 01 \ldots 0101 & * * * 101 \ldots 0101 \\
\left((-1)^{n+1} A^{-8}\langle L\rangle\right) & * 010101 \ldots 01 & 10101 \ldots 01 \\
\hline\left((-1)^{n+1}\left(1-A^{-8}\right)\langle L\rangle\right) & * * * \underbrace{00 \ldots 001}_{n} 1 & * * * \underbrace{00 \ldots 001}_{n} 1
\end{array}
$$

When $p=q=n$ in (2.1), we obtain the Jones polynomial of the $(n, n)$ torus link, which is the closure of the full twist in the braid group $B_{n}$, i.e., $\overline{\Delta_{n}^{2}}$ :

$$
\begin{aligned}
V_{\overline{\Delta_{n}^{2}}}(t) & =(-1)^{n+1} \frac{t^{\frac{1}{2}(n-1)^{2}}}{1-t^{2}} \sum_{i=0}^{n}\left(\begin{array}{c}
n \\
i
\end{array}\right) t^{(1+i)(n-i)}\left(t^{n-i}-t^{1+i}\right) \\
& =(-1)^{n+1} \frac{t^{\frac{1}{2}(n-1)^{2}}}{1-t^{2}}\left(1+(n-2) t^{n+1}+\{\text { higher order terms }\}\right) .
\end{aligned}
$$

The Kauffman bracket of $\overline{\Delta_{n}^{2}}$ is obtained from (2.2) by substituting $t=A^{-4}$ and, to adjust for the writhe, multiplying by $(-A)^{3 n(n-1)}=A^{3 n^{2}-3 n}$. The first two terms of $\left(1-t^{2}\right) \cdot V(t)$ change as follows:

$$
A^{3 n^{2}-3 n} \cdot A^{-2(n-1)^{2}}\left(1+(n-2) A^{-4(n+1)}\right)=A^{n^{2}+n-2}+(n-2) A^{n^{2}-3 n-6} .
$$

After dividing by $1-A^{-8}$ we obtain a sum that depends on the parity of $n$. We see that the Kauffman bracket of the positive full twist on $n$ strands has a gap block with top degree $n^{2}+n-2$, and its smallest nonzero coefficient has degree $n^{2}-3 n \pm 2$, according to whether $n$ is even or odd. However, when $n$ is odd, the 
zero "term" is the first one in the gap block, so we say that the gap block has bottom degree $n^{2}-3 n-2$, i.e.,

$$
(-1)^{n+1}\left\langle\overline{\Delta_{n}^{2}}\right\rangle=q_{2}(A)+a_{n} A^{n^{2}-3 n-2}+A^{n^{2}-3 n+2}+\cdots+A^{n^{2}+n-2},
$$

where $q_{2}(A)$ is a Laurent polynomial with degree strictly less than $n^{2}-3 n-2$ and $a_{n}=\left(1+(-1)^{n}\right) / 2$. We will show that adding a sufficiently positive braid $\beta^{\prime}$ to $\Delta_{n}^{2}$ does not affect the gap block.

The Temperley-Lieb algebra $T L_{n}$ is closely related to the Jones polynomial. In the usual notation, $T L_{n}$ is the algebra over $\mathbb{Z}\left[A^{ \pm 1}\right]$ with generators $\left\{\mathbf{1}, e_{1}, \ldots, e_{n-1}\right\}$ and relations, with $\delta=-A^{2}-A^{-2}$,

$$
e_{i}^{2}=\delta e_{i}, \quad e_{i} e_{i \pm 1} e_{i}=e_{i}, \quad e_{i} e_{j}=e_{j} e_{i} \text { if }|i-j| \geq 2 .
$$

As a free $\mathbb{Z}\left[A^{ \pm 1}\right]$-module, $T L_{n}$ has a basis that consists of all diagrams with no crossings and no closed curves, with dimension equal to the Catalan number $C_{n}=\frac{1}{n+1}\left(\begin{array}{c}2 n \\ n\end{array}\right)$. We will refer to this particular basis as the standard basis $\left\{h_{i} \mid i=\right.$ $\left.0, \ldots, C_{n}-1\right\}$ with $h_{0}=1$. Each $h_{i}$ can be expressed as a product of distinct generators: $h_{i}=e_{j_{1}} \ldots e_{j_{r}}$.

Let $A^{a-b}$ be the contribution from any smoothing $s$ of $\beta^{\prime}$ where $a$ and $b$ are the number of $A$ and $B$ smoothings of $\beta^{\prime}$, respectively. Passing to the representation of $\beta^{\prime}$ in $T L_{n}$,

$$
\beta^{\prime}=\sum_{s} A^{a(s)-b(s)} \bigcirc^{|s|} h_{i(s)},
$$

where $h_{i(s)}$ is the basis element obtained from a smoothing $s$, and $|s|$ is the number of loops in the smoothing of $\beta^{\prime}$ (not the closure of $\beta^{\prime}$ ).

Let $c=\operatorname{length}\left(\beta^{\prime}\right)$. For $0 \leq \ell \leq n$, the (unique) state which gives $h_{0}$ has $\ell$ $B$-smoothings, for which $a-b=(c-\ell)-\ell=c-2 \ell$. We define $q_{1}(A)$ as follows:

$$
\begin{aligned}
(-1)^{n+1} q_{1}(A) & =\left\langle\overline{\Delta_{n}^{2} \beta^{\prime}}\right\rangle-A^{c-2 \ell}\left\langle\overline{\Delta_{n}^{2} h_{0}}\right\rangle \\
& =\sum_{s \text { with } i(s) \neq 0} A^{a(s)-b(s)}\left\langle\bigcirc^{|s|} \overline{\Delta_{n}^{2} h_{i(s)}}\right\rangle \\
& =\sum_{s \text { with } i(s) \neq 0} A^{a(s)-b(s)} \delta^{|s|}\left\langle\overline{\Delta_{n}^{2} h_{i(s)}}\right\rangle .
\end{aligned}
$$

By Lemma 2.3 , which is proved below, for $i>0$ the highest power in $\left\langle\overline{\Delta_{n}^{2} h_{i}}\right\rangle$ is $n^{2}-3 n-4$. This implies the following:

Lemma 2.1. If $0 \leq \ell \leq n$, the degree of $q_{1}(A)$ is at most $c+n^{2}-3 n-6+2 \ell$.

Proof. First, suppose $\ell=0$. We claim that to get $k$ loops in any smoothing of $\beta^{\prime}$ we need at least $k+1 B$-smoothings. Since $\beta^{\prime}$ is a positive braid, every $B$-smoothing adds at most one loop, but the first $B$-smoothing does not result in any loops. Hence, $k+1 B$-smoothings (and the remaining $A$-smoothings) result in at most $k$ loops.

It follows that $1 \leq k+1 \leq b$ and $a \leq c-k-1$. Hence, $a-b \leq c-2 k-2$ and the highest power in $\delta^{k}$ is $2 k$. Thus, for $\ell=0$, the highest power in $A^{a(s)-b(s)} \delta^{|s|}$ is $c-2 k-2+2 k=c-2$.

If $\ell>0$, we claim that to get $k$ loops in any smoothing of $\beta^{\prime}$ we need at least $k+1-\ell B$-smoothings. As for a positive braid, $k+1$ smoothings that produce a cup-cap give at most $k$ loops. But now, some of these smoothings could be $A$ smoothings at a negative crossing, so $k+1-\ell B$-smoothings (and the remaining $A$-smoothings) result in at most $k$ loops. 
It follows that $b \geq k+1-\ell$ and $a \leq c-k-1+\ell$. Hence, $a-b \leq c-2 k-2+2 \ell$. Since the highest power in $\delta^{k}$ is $2 k$, the highest power in $A^{a(s)-b(s)} \delta^{|s|}$ is $c-2 k-$ $2+2 \ell+2 k=c+2 \ell-2$.

By Lemma 2.3. the highest power in $\left\langle\overline{\Delta_{n}^{2} h_{i(s)}}\right\rangle$ is $n^{2}-3 n-4$, so the degree of $q_{1}(A)$ is at most $c+n^{2}-3 n-6+2 \ell$.

We now return to the equation, $(-1)^{n+1} q_{1}(A)=\left\langle\overline{\Delta_{n}^{2} \beta^{\prime}}\right\rangle-A^{c-2 \ell}\left\langle\overline{\Delta_{n}^{2}}\right\rangle . \quad$ By Lemma 2.1, the degree of $q_{1}(A)$ is at most $\alpha_{1}=c+n^{2}-3 n-6+2 \ell$. Hence by (2.3),

$$
\begin{aligned}
(-1)^{n+1}\left\langle\overline{\Delta_{n}^{2} \beta^{\prime}}\right\rangle=q_{1}(A)+ & A^{c-2 \ell}\left(q_{2}(A)\right. \\
& \left.+a_{n} A^{n^{2}-3 n-2}+A^{n^{2}-3 n+2}+\cdots+A^{n^{2}+n-2}\right) .
\end{aligned}
$$

The highest power of $A$ above is $n^{2}+n-2+c-2 \ell$. Thus, the gap block starts at the power $\alpha_{2}=c+n^{2}-3 n-2-2 \ell$. Comparing with the maximum power of $q_{1}(A)$, we see that $q_{1}(A)$ and the gap block can overlap in at most $\ell$ coefficients: $\alpha_{1}-\alpha_{2}=4(\ell-1)$. After multiplying by $\left(1-t^{2}\right)$, we get a gap of $n-\ell$ zeros.

Let us compute the highest power of $A$ after adjusting for the writhe. The writhe $w=n(n-1)+(c-\ell)-\ell=n^{2}-n+c-2 \ell$. So after multiplying by $\left(-A^{3}\right)^{-w}$, the highest power of $A$ is

$$
(-1)^{n+1} A^{n^{2}+n-2+c-2 \ell}(-1)^{n^{2}-n+c-2 \ell} A^{-3\left(n^{2}-n+c-2 \ell\right)}=(-1)^{n+c+1}\left(A^{-4}\right)^{\frac{(n-1)^{2}+c-2 \ell}{2}} .
$$

To get the Jones polynomial, we substitute $t=A^{-4}$ and multiply by $\left(-A^{3}\right)^{-w}$. Hence we obtain the lowest power of $t$ to be $(-1)^{n+c+1} t^{\frac{(n-1)^{2}+c-2 \ell}{2}}$. This completes the proof of the first statement of Theorem 1.1

To obtain the tail (without denominators), we note that $\frac{1+t p(t)}{1-t^{2}}=1+t q(t)$, where $q(t)$ is a polynomial. For simplicity, let $\ell=0$, but the proof is the same in the other case. Given the polynomial $p(t)$ from the proof above, we obtain the polynomial $q(t)$ as follows:

$$
n \text { odd }
$$

$$
\begin{aligned}
\frac{1+t^{n+1} p(t)}{1-t^{2}} & =\frac{1-t^{n+1}}{1-t^{2}}+\frac{t^{n+1}(1+p(t))}{1-t^{2}} \\
& =\sum_{i=0}^{[n / 2]} t^{2 i}+t^{n+1} q(t),
\end{aligned}
$$

$n$ even

$$
\begin{aligned}
\frac{1+t^{n+1} p(t)}{1-t^{2}} & =\frac{1-t^{n}}{1-t^{2}}+\frac{t^{n}(1+t p(t))}{1-t^{2}} \\
& =\sum_{i=0}^{(n-2) / 2} t^{2 i}+t^{n}(1+t q(t)) \\
& =\sum_{i=0}^{n / 2} t^{2 i}+t^{n+1} q(t) .
\end{aligned}
$$

This completes the proof of Theorem 1.1.

Lemma 2.2. Let $h_{i}$ be a standard basis element of $T L_{n}$. Let $k=\#$ cups in $h_{i}$, and $m=\#$ through strands in $h_{i}$, so that $2 k+m=n$. If $H_{i}=h_{i}$ with its $m$ through 


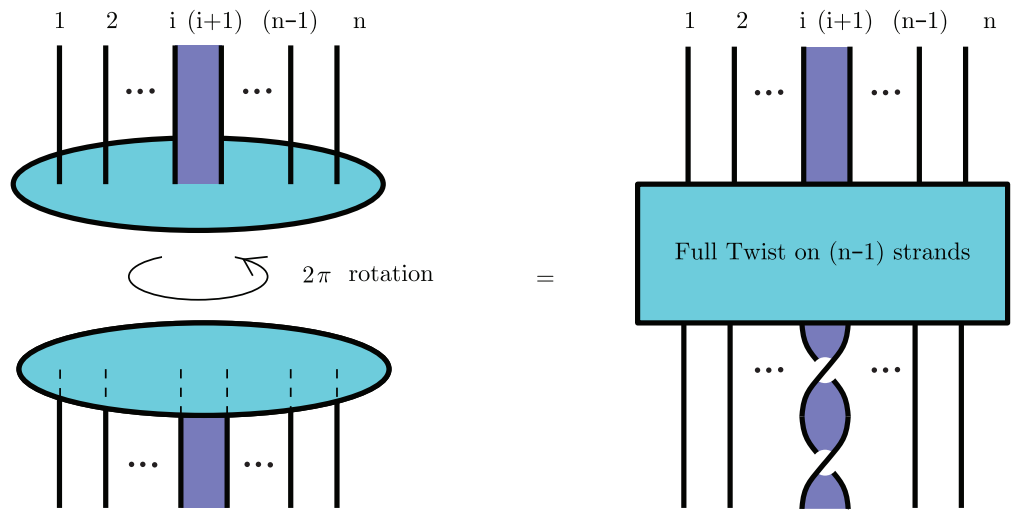

FiguRE 1. Full twist on $n$ strands, with two consecutive strands grouped together as one strand.
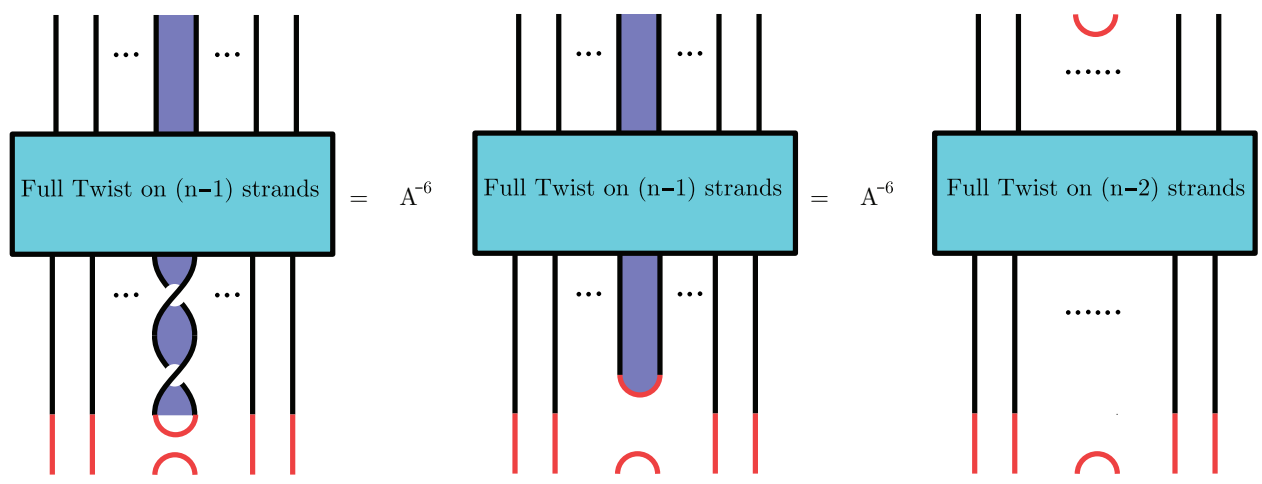

Figure 2. $\Delta_{n}^{2} e_{i}=A^{-6} E_{i}$

strands given a full right twist, then $\Delta_{n}^{2} h_{i}=\Delta_{n}^{2}$ if $i=0$, and $\Delta_{n}^{2} h_{i}=A^{-6 k} H_{i}$ if $i>0$.

Proof. Let $B=\{1,2, \ldots, 2 n\}$ denote the positions of the strands. Let $\operatorname{cap}(h)=$ $\left\{u_{1}, \ldots, u_{2 k}\right\}$ denote indices of strands of $h$ which are caps, e.g., cap $\left(e_{i}\right)=\{i, i+1\}$. Let feet $(h)=\left\{v_{1}, \ldots, v_{m}\right\}$ denote the bottom indices of strands of $h$ which pass through. Note that $\operatorname{cap}(h) \sqcup$ feet $(h)=B$.

We will prove the claim by induction on the length of $h$ as a product of $e_{i}$ 's. For the base case, $h=e_{i}$. If $E_{i}$ denotes $e_{i}$ with its $n-2$ through strands given a full right twist, then from Figures [1] and [2] we see that $\Delta_{n}^{2} e_{i}=A^{-6} E_{i}$.

Assuming the claim holds for $h^{\prime}=e_{i_{1}} \ldots e_{i_{r-1}}$, we must show that it holds for $h=h^{\prime} e_{i_{r}}$. For the standard $T L_{n}$ basis, we have that $i_{r}$ is distinct from $i_{1}, \ldots, i_{r-1}$, so that $\operatorname{cap}\left(e_{i_{r}}\right) \nsubseteq \operatorname{cap}\left(h^{\prime}\right)$. This gives us two cases (see Figure 3):

Case 1. $\operatorname{cap}\left(e_{i_{r}}\right) \subseteq$ feet $\left(h^{\prime}\right)$. In this case, $|\operatorname{cap}(h)|=\left|\operatorname{cap}\left(h^{\prime}\right)\right|+2$. The full twist on $\mid$ feet $\left(h^{\prime}\right) \mid$ strands is paired with $e_{i_{r}}$ and results in $A^{-6} \Delta_{\mid \text {feet }\left(h^{\prime}\right) \mid-2}^{2}$. Hence, $\Delta_{n}^{2} h=$ $A^{-6(k-1)} H^{\prime} e_{i_{r}}=A^{-6 k} H$. 


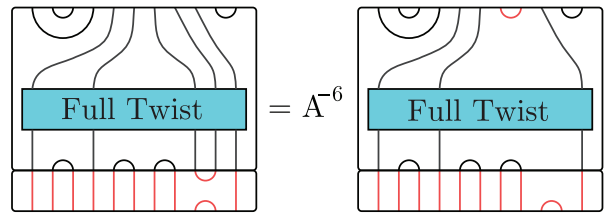

Case 1

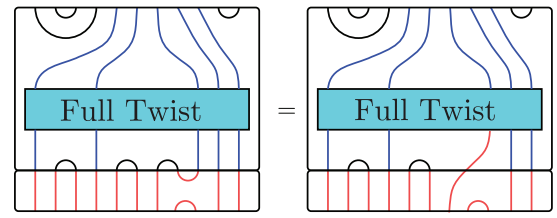

Case 2

Figure 3. Two cases for Lemma 2.2

Case 2. $\operatorname{cap}\left(e_{i_{r}}\right) \nsubseteq$ feet $\left(h^{\prime}\right)$. In this case, $\operatorname{cap}\left(e_{i_{r}}\right)$ is split between feet $\left(h^{\prime}\right)$ and $\operatorname{cap}\left(h^{\prime}\right)$. The number of caps and the number of through strands of $h$ are both the same as those in $h^{\prime}$. Hence, $\Delta_{n}^{2} h=A^{-6 k} H^{\prime} e_{i_{r}}=A^{-6 k} H$.

Lemma 2.3. The highest power of $A$ in $\left\langle\overline{\Delta_{n}^{2} h_{i}}\right\rangle$ for any $i>0$ is less than or equal to $n^{2}-3 n-4$.

Proof. Following the notation in Lemma 2.2, $k=$ \#cups in $h_{i}$, so that $1 \leq k \leq$ $[n / 2]$. By Lemma 2.2, $\Delta_{n}^{2} h_{i}=A^{-6 k} H_{i}$ if $i>0$, where $H_{i}$ also has $k$ cups. The closure of $H_{i}$ will result in $k^{\prime}$ cups paired with caps to produce loops and $k^{\prime \prime}$ cups pulled through the full twist with a factor of $A^{-6 k^{\prime \prime}}$, where $0 \leq k, k^{\prime \prime} \leq k$. So $\overline{H_{i}}$ will have a full twist on $m^{\prime}$ strands, where $0 \leq m^{\prime} \leq m$. Thus, $\left\langle\overline{\Delta_{n}^{2} h_{i}}\right\rangle=A^{-6 k}\left\langle\overline{H_{i}}\right\rangle=$ $A^{-6\left(k+k^{\prime \prime}\right)} \delta^{k^{\prime}}\left\langle\overline{\Delta_{m^{\prime}}^{2}}\right\rangle$.

The highest power of $A$ is $\left(-6 k-6 k^{\prime \prime}+2 k^{\prime}+\left(m^{\prime}\right)^{2}+m^{\prime}-2\right)$, which is maximized when $k^{\prime \prime}=0, k^{\prime}=k$ and $m^{\prime}=m$. Since $2 k+m=n$, we have $m^{2}+m-2-4 k=$ $(n-2 k)^{2}+(n-2 k)-2-4 k$. The function $f(k)=(n-2 k)^{2}+(n-2 k)-2-4 k$ has an absolute minimum at $k=\frac{n}{2}+\frac{3}{4}$ and is decreasing for $k<\frac{n}{2}+\frac{3}{4}$. Since $1 \leq k \leq[n / 2]<\frac{n}{2}+\frac{3}{4}, f(k) \leq f(1)=(n-2)^{2}+(n-2)-2-4=n^{2}-3 n-4$.

\section{Proof of Theorem 1.3}

For any link diagram $D$, let $D^{(r)}$ denote its blackboard framed $r$-cable. Let $D_{n}$ denote the standard diagram of the closure of a full positive twist on $n$ strands with a positive kink on each strand.

Lemma 3.1. $D_{n}^{(r)}=D_{n r}$.

Proof. The belt trick (see, e.g., §§2.2-2.4 of [16]) implies:

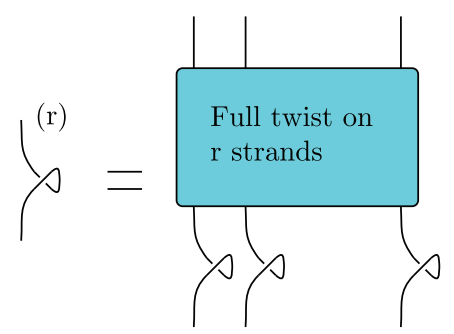

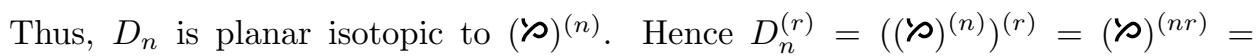
$D_{n r}$. 
For $N \geq 0$, let $S_{N}(x)$ be the Chebyshev polynomials defined by

$$
S_{N}(x)=\sum_{j=0}^{[N / 2]}(-1)^{j}\left(\begin{array}{c}
N-j \\
j
\end{array}\right) x^{N-2 j} .
$$

For a link diagram $D$, let $S_{N}(D)$ be a linear combination of blackboard cablings of $D$. We can define the colored Jones polynomial, as in equation (1.1), by a related expression in terms of the blackboard framing for $D$ (see, e.g., 6]):

$$
J_{N+1}\left(D ; A^{-4}\right)=\left((-1)^{N} A^{N^{2}+2 N}\right)^{-w(D)}(-1)^{N-1}\left(A^{2}+A^{-2}\right)\left\langle S_{N}(D)\right\rangle .
$$

As before, let $\beta^{\prime}$ be a positive $n$-braid with $c$ crossings, $\beta=\Delta_{n}^{2} \beta^{\prime}$, and $L=\bar{\beta}$. Let $D$ be a diagram of $L$ given by the closure of $\beta$ with a positive kink on every strand after the full twist. Note that the writhe of $D, w(D)=n^{2}+c$. By Lemma3.1. $D^{(r)}$ is the closure of the braid $\Delta_{n r}^{2} \beta^{\prime(r)}$ with a positive kink on every strand following the full twist.

Let $r(A)=(-1)^{N(w(D)+1)+1} A^{-\left(N^{2}+2 N\right) w(D)}\left(A^{2}+A^{-2}\right)$. By equation (3.1),

$$
\begin{aligned}
\left(1-A^{-8}\right) J_{N+1}\left(L ; A^{-4}\right)=(1- & \left.A^{-8}\right) r(A) \\
& \times \sum_{j=0}^{[N / 2]}(-1)^{j}\left(\begin{array}{c}
N-j \\
j
\end{array}\right)\left\langle D^{(N-2 j)}\right\rangle \\
=r(A) & \sum_{j=0}^{[N / 2]}(-1)^{j}\left(\begin{array}{c}
N-j \\
j
\end{array}\right)\left(-A^{3}\right)^{n(N-2 j)} \\
& \times\left(1-A^{-8}\right)\left\langle\frac{\left.\Delta_{n(N-2 j)}^{2} \beta^{\prime(N-2 j)}\right\rangle}{}\right.
\end{aligned}
$$

By equation (2.4) with $\ell=0$,

$$
\begin{aligned}
\left(1-A^{-8}\right)\left\langle\overline{\Delta_{n}^{2} \beta^{\prime}}\right\rangle & =(-1)^{n+1}\left(q_{3}(A)+(n-2) A^{n^{2}-3 n-6+c}+A^{n^{2}+n-2+c}\right) \\
& =(-1)^{n+1}\left(q_{4}(A) A^{n^{2}-3 n-6+c}+A^{n^{2}+n-2+c}\right),
\end{aligned}
$$

where $q_{3}(A)$ and $q_{4}(A)$ are Laurent polynomials such that $\max \operatorname{deg}\left(q_{3}(A)\right) \leq n^{2}-$ $3 n-6+c$ and $\max \operatorname{deg}\left(q_{4}(A)\right) \leq 0$.

Let $d_{1}(j)=(N-2 j)^{2}\left(n^{2}+c\right)-6$ and $d_{2}(j)=(N-2 j)^{2}\left(n^{2}+c\right)+4 n(N-2 j)-2$. Note that $d_{1}(j)$ and $d_{2}(j)$ are both quadratic functions of $j$. As $j$ increases from 0 to $[N / 2]$, they decrease and $d_{2}(j)>d_{1}(j)$.

Lemma 3.2. $d_{1}(j)>d_{2}(j+1)$ for $0 \leq j \leq[N / 2]-1$.

Proof. Note that $n \geq 3$ and $N \geq 3$. Then

$$
\begin{aligned}
d_{1}(j)-d_{2}(j+1)= & \left((N-2 j)^{2}\left(n^{2}+c\right)-6\right) \\
& -\left((N-2 j-2)^{2}\left(n^{2}+c\right)+4 n(N-2 j-2)-2\right) \\
= & 4(N-2 j)\left(n^{2}+c\right)-4\left(n^{2}+c\right)-4 n(N-2 j-2)-4 \\
= & 4 n(n(N-2 j)-(N-2 j)-n)+4 c(N-2 j-1)+8 n-4 \\
= & 4 n(n-1)(N-2 j-1)+4 c(N-2 j-1)+4(n-1) \\
> & 0 \text { for } 0 \leq j \leq[N / 2]-1 .
\end{aligned}
$$


Let $b_{j}=(-1)^{j}\left(\begin{array}{c}N-j \\ j\end{array}\right)$. Continuing from (3.2),$\left(1-A^{-8}\right) J_{N+1}\left(L ; A^{-4}\right)$

$$
\begin{aligned}
& =r(A) \sum_{j=0}^{[N / 2]} b_{j}\left(-A^{3}\right)^{n(N-2 j)} \times\left(1-A^{-8}\right)\left\langle\overline{\Delta_{n(N-2 j)}^{2} \beta^{\prime(N-2 j)}}\right\rangle \\
& =(-1)^{n N} r(A) \sum_{j=0}^{[N / 2]}(-1)^{n(N-2 j)+1} b_{j}\left[\bar{p}_{N-2 j}(A) A^{d_{1}(j)}+A^{d_{2}(j)}\right] \quad \text { using (3.3) } \\
& =-r(A) \sum_{j=0}^{[N / 2]} b_{j}\left[A^{d_{1}(j)} \bar{p}_{N-2 j}(A)+A^{d_{2}(j)}\right] \\
& =-r(A)\left[A^{d_{1}(0)} p_{N}(A)+A^{d_{2}(0)}\right] \text { using Lemma 3.2. }
\end{aligned}
$$

where $p_{N}(A)$ and $\bar{p}_{N-2 j}(A)$ are Laurent polynomials such that $\max \operatorname{deg} p_{N}(A) \leq 0$ and $\max \operatorname{deg}\left(\bar{p}_{N-2 j}(A)\right) \leq 0$ for $0 \leq j \leq[N / 2]$, respectively.

Substituting $t=A^{-4}$ and, in $r(A), w(D)=n^{2}+c$ :

$$
\begin{aligned}
& \left(1-t^{2}\right) J_{N+1}(L ; t)=(-1)^{-N\left(n^{2}+c-1\right)} t^{\frac{\left(N^{2}+2 N\right)\left(n^{2}+c\right)}{4}}\left(t^{1 / 2}+t^{-1 / 2}\right) \\
& \times\left(t^{-d_{2}(0) / 4}+t^{-d_{1}(0) / 4} p_{N}(t)\right) \\
& =(-1)^{-N\left(n^{2}+c-1\right)}\left(t^{1 / 2}+t^{-1 / 2}\right) \\
& \times\left(t^{\frac{N\left(n^{2}+c-2 n\right)+1}{2}}+t^{\frac{N\left(n^{2}+c\right)+3}{2}} p_{N}(t)\right), \\
& (1-t) J_{N+1}(L ; t)=(-1)^{N\left(n^{2}+c-1\right)} t^{\frac{N\left(n^{2}+c-2 n\right)}{2}}\left(1+t^{n N+1} p_{N}(t)\right) \text {, }
\end{aligned}
$$

where $p_{N}(t)$ is a polynomial. Now, the statement of Theorem 1.3 follows from

$$
J_{N}^{\prime}(L ; t)=\frac{J_{N}(L ; t)}{[N]}=\frac{t^{\frac{N-1}{2}}(1-t) J_{N}(L ; t)}{\left(1-t^{N}\right)} .
$$

To obtain the tail (without denominators), we suppress $\bar{\beta}$ in the notation:

$$
\begin{aligned}
\frac{1+t^{n N+1} p_{N}(t)}{1-t} & =\frac{1-t^{n N+1}}{1-t}+\frac{t^{n N+1}\left(1+p_{N}(t)\right)}{1-t} \\
& =\sum_{i=0}^{n N} t^{i}+t^{n N+1} q_{N}(t) \\
\frac{1+t^{n N+1} p_{N}(t)}{1-t^{N+1}} & =\frac{1-t^{n(N+1)}}{1-t^{N+1}}+\frac{t^{n N+1}\left(t^{n-1}+p_{N}(t)\right)}{1-t^{N+1}} \\
& =\sum_{i=0}^{n-1} t^{i(N+1)}+t^{n N+1} q_{N}(t) .
\end{aligned}
$$

This completes the proof of Theorem 1.3 


\section{REFERENCES}

[1] C. Armond. Walks along braids and the colored Jones polynomial, arXiv:1101.3810v1 [math.GT], 2011.

[2] J. Birman and I. Kofman. A new twist on Lorenz links. J. Topol., 2(2):227-248, 2009. MR2529294(2010i:57010)

[3] A. Champanerkar, D. Futer, I. Kofman, W. Neumann, and J. Purcell. Volume bounds for generalized twisted torus links, Math. Res. Lett., 18(6):1097-1120, 2011. MR2915470

[4] A. Champanerkar and I. Kofman. On the Mahler measure of Jones polynomials under twisting. Algebr. Geom. Topol., 5:1-22, 2005. MR2135542(2006b:57010)

[5] A. Champanerkar, I. Kofman, and E. Patterson. The next simplest hyperbolic knots. J. Knot Theory Ramifications, 13(7):965-987, 2004. MR2101238 (2005k:57010)

[6] O. Dasbach and X.-S. Lin. On the head and the tail of the colored Jones polynomial. Compos. Math., 142(5):1332-1342, 2006. MR2264669 (2007g:57018)

[7] J. Franks and R. F. Williams. Braids and the Jones polynomial. Trans. Amer. Math. Soc., 303(1):97-108, 1987. MR896009 (88k:57006)

[8] D. Futer, E. Kalfagianni, and J. Purcell. Dehn filling, volume, and the Jones polynomial. J. Differential Geometry, 78(3):429-464, 2008. MR2396249 (2009c:57010)

[9] D. Futer, E. Kalfagianni, and J. Purcell. Cusp areas of Farey manifolds and applications to knot theory. Int. Math. Res. Not. IMRN, 23:4434-4497, 2010. MR2739802 (2011k:57027)

[10] S. Garoufalidis. The degree of a $q$-holonomic sequence is a quadratic quasi-polynomial. Electron. J. Combin., 18(2):Paper 4, 23, 2011. MR2795781

[11] J.-M. Isidro, J. M. F. Labastida, and A. V. Ramallo. Polynomials for torus links from ChernSimons gauge theories. Nuclear Phys. B, 398(1):187-236, 1993. MR1222806 (95e:57014)

[12] V. Jones. Hecke algebra representations of braid groups and link polynomials. Ann. of Math. (2), 126(2):335-388, 1987. MR908150 (89c:46092)

[13] R. Kirby and P. Melvin. The 3-manifold invariants of Witten and Reshetikhin-Turaev for sl(2, C). Invent. Math., 105(3):473-545, 1991. MR1117149(92e:57011)

[14] H. R. Morton. Seifert circles and knot polynomials. Math. Proc. Cambridge Philos. Soc., 99(1):107-109, 1986. MR809504 (87c:57006)

[15] A. Stoimenow. On the crossing number of positive knots and braids and braid index criteria of Jones and Morton-Williams-Franks. Trans. Amer. Math. Soc., 354(10):3927-3954 (electronic), 2002. MR 1926860 (2003f:57022)

[16] H. Wenzl. Braids and invariants of 3-manifolds. Invent. Math., 114(2):235-275, 1993. MR $1240638(94 \mathrm{i}: 57021)$

Department of Mathematics, College of Staten Island, City University of New York, Staten Island, New York 10314 - and - Department of Mathematics, Graduate Center, City University of New York, 365 Fifth Avenue, New York, New York 10016

E-mail address: abhijit@math.csi.cuny.edu

Department of Mathematics, College of Staten Island, City University of New York, Staten Island, New York 10314 - and - Department of Mathematics, Graduate Center, City University of New York, 365 Fifth Avenue, New York, New York 10016

E-mail address: ikofman@math.csi.cuny.edu 